\title{
Eine Smartphone-App für einen nachhaltigen Lebensstil
}

\author{
Mobile Endgeräte wie Smartphones drängen \\ auf den Massenmarkt. Mit der ständigen \\ Datenverfügbarkeit und innovativen Applikationen \\ sind auch Möglichkeiten zur Förderung von \\ nachhaltigen Lebensstilen verbunden. Es gibt \\ bereits erste Smartphone-Apps zum Thema \\ ökologische Nachhaltigkeit. \\ Von Frank Heidmann, Judith Bauer und \\ Alexander Warth
}

D er Einsatz moderner Informations- und Kommunikationstechnologien (IKT) zur Förderung individueller Einstellungs- und Verhaltensänderungen hinsichtlich nachhaltiger Lebensstile ist in den letzten Jahren $\mathrm{zu}$ einem wichtigen Anwendungsfeld für Green IT geworden (Tomlinson 2010). Dabei können zwei grundlegende Funktionen von IKT unterschieden werden: Auf der einen Seite bietet der Computer vielfältige Optionen der Visualisierung von Umwelt-, Wirtschafts- oder Konsumphänomenen mit dem Ziel, komplexe Zusammenhänge verständlich, emotional berührend und immer häufiger auch ästhetisch anspruchsvoll sichtbar zu machen. Ein gutes Beispiel hierfür ist das interaktive Buch „Our Choice“ von Al Gore (2011).

Auf der anderen Seite unterstützt der Computer die Kommunikation innerhalb der Gesellschaft. Soziale Netze wie Facebook helfen, Ideen zu verbreiten, erleichtern die schnelle Gründung von Interessengruppen, gestatten soziale Unterstützung aber auch Sanktionen. Beide Funktionen von IKT sollen den Nutzer bei der Entscheidungsfindung im Kontext nachhaltigen Konsums unterstützen und Anstöße zur Veränderung mentaler Modelle geben. Mobile Endgeräte wie Smartphones spielen dabei eine immer größere Rolle. Sie weisen einige Charakteristika auf, die sie für Problemlösungen hin zu einem nachhaltigen Lebensstil scheinbar besonders prädestinieren, beispielsweise den allgegenwärtigen Zugriff auf Informationen unabhängig von Raum und Zeit (Pitt et al. 2011). So ist es nicht verwunderlich, dass immer mehr Applikationen mit Inhalten und Funktionen im Kontext ökologischer Nachhaltigkeit in den Online-Shops zu finden sind.
Unter diesen Maßgaben ist Anfang 2011 die iPhone-App EcoChallenge entwickelt worden. Idee der App ist es, den Nutzer durch wöchentliche Informationen und entsprechende Aufgabenstellungen, die sogenannten Challenges, zu neuen Aspekten nachhaltigen Lebens zu motivieren. Um die Verhaltensänderung der Nutzer zu fördern, sind das hoch ästhetische Erscheinungsbild sowie ihre mühelose Nutzbarkeit wichtig, deren Einflusswirkung bereits in vielen Zusammenhängen nachgewiesen wurde (Moshagen/Thielsch 2010) und gerade für die Akzeptanz von Nachhaltigkeitsthemen unumgänglich erscheint (Bittencourt et al. 2003).

\section{Die EcoChallenge-App}

Im Bereich „Thema“ werden redaktionell aufbereitete Informationen, zum Beispiel zur Verwendung von Kunststoffen, in Form von Infografiken nachvollziehbar visualisiert. Dem Nutzer wird so zunächst ein schnelles und einfaches Erfassen relevanter Informationen ermöglicht. Durch einen „Rechner“ werden hieran die vermittelten Informationen in Relation zu persönlichen Lebensgewohnheiten gesetzt, beispielsweise wie hoch der eigene Fleischkonsum ausfällt, und durch zwei „Challenges“ pro Woche eine entsprechende spielerische Auseinandersetzung mit nachhaltigen Themen forciert. Derartige Vermittlungsketten und selbstgesteuertes Lernen verstärken effizient die Gedächtnisleistung (Brandt 2008). Das spielerische Element motiviert, das Vermittelte in konkreten Zusammenhang zu eigenen Alltagshandlungen zu bringen, etwa Freunde zu einem Abendessen mit $\rightarrow$
Abbildung 1: Screenshots der EcoChallenge-App

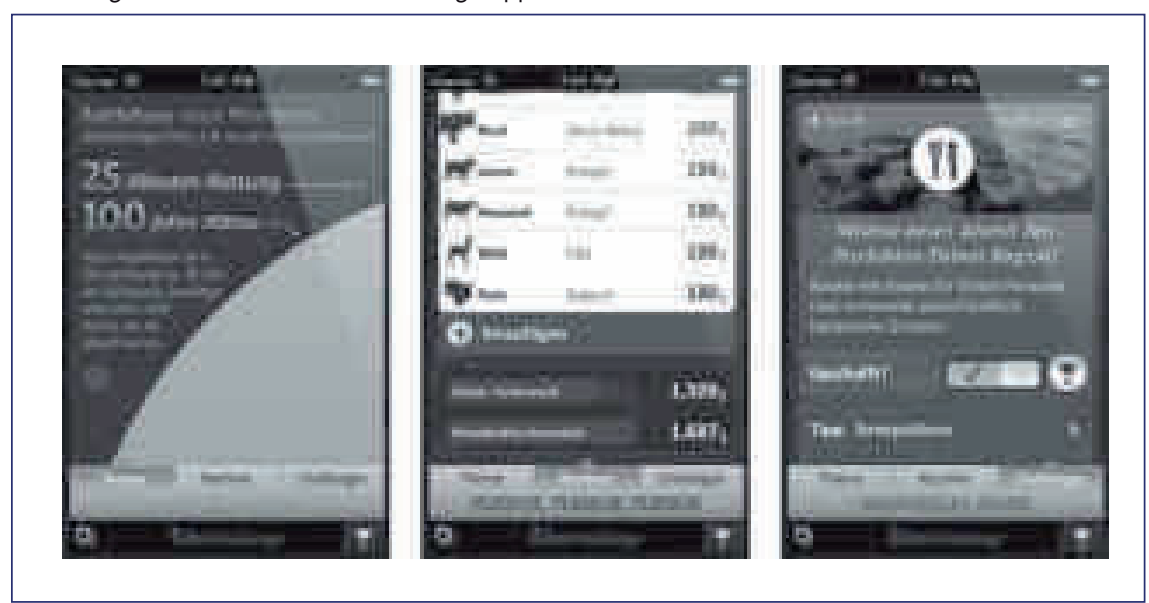

Quelle: raureif $\mathrm{GmbH}$ 
regionalen Zutaten einzuladen. Derartiges „Learning by Doing and Learning by Thinking“ kann zu eindrucksvollen Verhaltensänderungen führen (Ericsson et al. 2006). Das Bewältigen der Challenges wird durch positive Rückmeldungen in Form digitaler Auszeichnungen belohnt und soll zudem ihre Ausführung anregen (Koger/Du Nann Winter 2010). Mit ihren individuellen Erfolgen können die Nutzer über Facebook in einem sozialen Netzwerk partizipieren, wodurc men ins Spiel kommt: Wenn Personen der eigenen Peer Group teilnehmen, steigt die Wahrscheinlichkeit, dass man dem Einfluss der Gruppe folgt (Schwartz 1973; Homburg/Matthies 1998).

Grundsätzlich legt die Verknüpfung verständlicher Information, spielerischer Aufgaben, sozialer Interaktion und ästhetischen Anspruchs die Vermutung nahe, dass die EcoChallenge ein wirkungsvolles Instrument hinsichtlich individueller Verhaltensänderung darstellt. Ob sie diesen Erwartungen gerecht wird, wurde anhand einer experimentellen Wirkstudie überprüft.

\section{Wirkliche Verhaltensänderungen?}

Zur Überprüfung, wie stark die beschriebenen Charakteristika wie Ästhetik und Alltagsintegration der iPhone-App auf Nutzer wirken, wurden drei verschiedene Experimentalgruppen definiert und mit einer Kontrollgruppe verglichen. Die iPhoneGruppe testete vier Wochen die EcoChallenge; äquivalente Informationen und Aufgaben wurden der Smartphone-Gruppe per E-Mail auf deren Smartphones sowie der E-Mail-Gruppe an den PC gesendet; die Kontrollgruppe erhielt keinerlei Informationen oder Aufgaben.

Die iPhone-Gruppe bewertete ästhetische Gestaltung und Benutzerfreundlichkeit hoch, verbrachte weit mehr Zeit mit der App und führte doppelt so viele Challenges aus wie die Smartphone- und E-Mail-Gruppe. Alle Experimentalgruppen zusammen genommen zeigten direkt nach den Interventionen einen signifikanten Anstieg im vermittelten Wissen und entsprechenden Einstellungen im Vergleich zur Kontrollgruppe. Allerdings war bereits nach zehn Wochen und erneuter Überprüfung kein signifikanter Unterschied mehr messbar. Die Verhaltensweisen änderten sich zu keinem Zeitpunkt in bedeutsamem Maße. Die iPhone-Gruppe zeigte im direkten Vergleich mit E-Mail- und Smartphone-Gruppe kaum Überlegenheit. Allein im Wissenstest schnitten die iPhone-Nutzer tendenziell besser ab.

\section{Fazit}

Die EcoChallenge schafft viele Voraussetzungen, die Einstellungs- und Verhaltensänderungen vermuten lassen. Dennoch kommt es zu keinem messbaren Erfolg. Wie ist das zu erklären? Mit vier Wochen wurde nur sehr kurz interveniert, auch Facebook wurde kaum genutzt. Weiterführend zu hinterfragen respektive zu erforschen wäre, ob die EcoChallenge nur als Spiel konsumiert wird und dadurch an Wirkung einbüßt.

Was die EcoChallenge jedoch eindrucksvoll aufzeigt, ist, dass sich Menschen zu forcierter Auseinandersetzung mit nachhal-

tigen Themen motivieren lassen. Ein erster Schritt in Richtung nachhaltiges Bewusstsein ist mit dieser Art an Apps erzielbar. Für Produkte, welche Menschen helfen sollen sich zu verändern, bedeutet es, dass diese Anwendungen durchaus ein Einstieg in ein bewusstes, nachhaltiges Leben sein können. Doch grundlegende Entscheidungen, die Suche nach Lebensalternativen und politische Partizipation erfordern die Entwicklung weiterer, viel tiefgreifenderer Interventionen im Alltag und Leben der Menschen und nicht nur auf dem Gebiet der Green IT.

\section{Literatur}

Bittencourt, I. / Borner, J. / Heiser, A.: Nachhaltigkeit in 50 Sekunden. Kommunikation für die Zukunft. München 2003.

Ericsson, K. A. / Charness, N. / Feltovich, P. J. / Hoffman, R. R.: The Cambridge Handbook of Expertise and Expert Performance. New York 2006.

Gore, A.: Our choice: A plan to solve the climate crisis (Book/App). New York 2009/2011.

Brandt, P.: Von der Effizienz zur Effektivität. Im Gespräch mit Joachim Hasebrook. In: DIE Zeitschrift für Erwachsenenbildung, 1/2008. S.26.

Homburg, A. / Matthies, E.: Umweltpsychologie: Umweltkrise, Gesellschaft und Individuum. Weinheim 1998.

Koger, S. M. / Du Nann Winter, D.: The psychology of environmental problems. New York 2010.

Moshagen, M. / Thielsch, M. T.: Facets of visual aesthetics. In: International Journal of Human Computer Studies, 68/2010. S.689-709.

Pitt, L.F. / Parent, M. / Junglas, I. / Chan, A. / Spyropoulou, S.: Integrating the smartphone into a sound environmental information systems strategy: Principles, practices and a research agenda. In: Journal of Strategic Information Systems, 20/2010. S.27-37.

Schwartz, S. H.: Normative explanations of helping behavior: A critique, proposal, and empirical test. In: Journal of Experimental Social Psychology, 9/1973, S.349-364.

Tomlinson, B.: Greening through IT. Cambridge. Massachusetts 2010.

\section{AUTOREN + KONTAKT}

Dr. Frank Heidmann ist Professor für Interface-Design an der Fachhochschule Potsdam.

Fachhochschule Potsdam, Pappelallee 8-9, 14469 Potsdam. Tel.: +493315801401,

E-Mail: heidmann@fh-potsdam.de

Dipl.-Psych. Judith Bauer promoviert im Fachbereich Nachhaltigkeitswissenschaften an der Leuphana Universität Lünebeburg.

Dipl.-Des. Alexander Warth ist wissenschaftlicher Mitarbeiter an der Fachhochschule Potsdam.
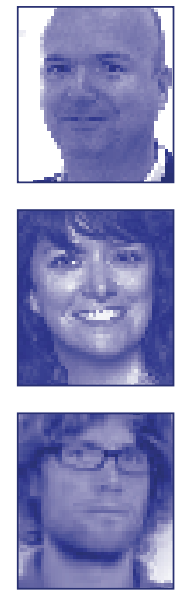


\section{Lizenzhinweis}

Die Beiträge in ÖkologischesWirtschaften werden unter der Creative-Commons-Lizenz "CC 4.0 Attribution Non-Commercial No Derivatives" veröffentlicht. Im Rahmen dieser Lizenz muss der Autor/Urheber stets genannt werden, das Werk darf nicht bearbeitet, abgewandelt oder in anderer Weise verändert und außerdem nicht kommerziell genutzt werden. Die digitale Version des Artikels bleibt für zwei Jahre Abonnent/innen vorbehalten und ist danach im Open Access verfügbar. 